\title{
Rapid Crystallization of Levitated and Undercooled Semiconducting Material Melts
}

\author{
YUSUKE ISHIBASHI, ${ }^{1,2}$ KAZUHIKO KURIBAYASHI, ${ }^{1}$ \\ and KATSUHISA NAGAYAMA ${ }^{1}$ \\ 1.-Shibaura Institute of Technology, Toyosu, Koto-ku, Tokyo 135-8548, Japan. 2.-e-mail: \\ mb11006@sic.shibaura-it.ac.jp
}

\begin{abstract}
Using a $\mathrm{CO}_{2}$ laser-equipped electromagnetic levitator, we carried out the containerless crystallization of $\mathrm{Si}$ and Ge. From the point of interface morphologies, the relation between growth velocities and undercoolings was classified into three regions. In regions I and II, although the morphologies of growing crystals are different: plate-like needle crystals in region I and facetted dendrite at region II, the growth velocities in these two regions are fundamentally scaled by the thermal diffusivities and the temperature increase caused by the release of the latent heat. This result means that the growth velocity can be expressed by the product of the thermal diffusivity and the growth kinetics. An analysis of the dendrite morphologies revealed that the kinetics of crystal growth in regions I and II represent two-dimensional nucleation at the reentrant corner formed at the edge of the two parallel twins. In region III, thermal diffusion-controlled interface attachment kinetics control as described by a modified Wilson-Frenkel model.
\end{abstract}

\section{INTRODUCTION}

In recent years, with advances in levitation techniques, research on solidification into the undercooled melt ${ }^{1,2}$ has brought new insight into the formation of metastable structures. Also, with the increase of solar power utilization, industry has shown interest in developing a process to form spherical crystals of Si for solar cell applications. ${ }^{3}$ In order to manufacture the spherical crystals, droptube processing is commonly employed as the massproduction method of choice. Here, the melt is ejected into a drop tube and the stream breaks up into numerous droplets that then solidify during free fall. This process is very simple; however, the yield of high-quality crystals that are suitable for use in solar cells is much lower than that achieved using conventional crystal growth techniques such as Czochraiski (CZ) and Bridgeman methods. The reason for this disadvantage can be simply attributed to the fact that the nucleation and growth of crystals during free fall cannot be monitored. In order to solve this problem, Aoyama, Takamura, and Kuribayashi (hereafter referred to as ATK) ${ }^{4}$ carried out a series of experiments using an electromagnetic levitation furnace equipped with a high-speed video camera, and they elucidated that the crystallization process is distinguished into three regions: In region I, which is under relatively low undercooling less than $100 \mathrm{~K}$, plate-like needle crystals appear. In region II under medium undercooling, from $100 \mathrm{~K}$ to $200 \mathrm{~K}$, facetted dendrites appear. In region III larger than $200 \mathrm{~K}$, the crystalmelt interface macroscopically looks flattened, indicating that the microstructure was significantly refined. In addition, analyzing the relation between undercooling $\Delta T$ and the growth velocity $V$ on the basis of a dendrite growth model incorporating linear kinetics, ${ }^{5}$ ATK reported that the kinetic coefficient $\mu$ is $0.4 \mathrm{~m} / \mathrm{sK}$. However, Watanabe, Nagayama, and Kuribayashi (WNK), ${ }^{6}$ based on precise measurement of the crystallization process for levitated droplets, reported that the crystal growth kinetics in regions I and II are not a linear function, but rather they showed an exponential increase, thus implying that the rate-controlling kinetics are not interface attachment but rather two-dimensional (2-D) nucleation. There remain inconsistencies in that the morphology of the crystal-melt interface in region $\mathrm{I}$ is quite different from that in region II. Furthermore the relation between $\Delta T$ and $V$, particularly in region II, appears too 


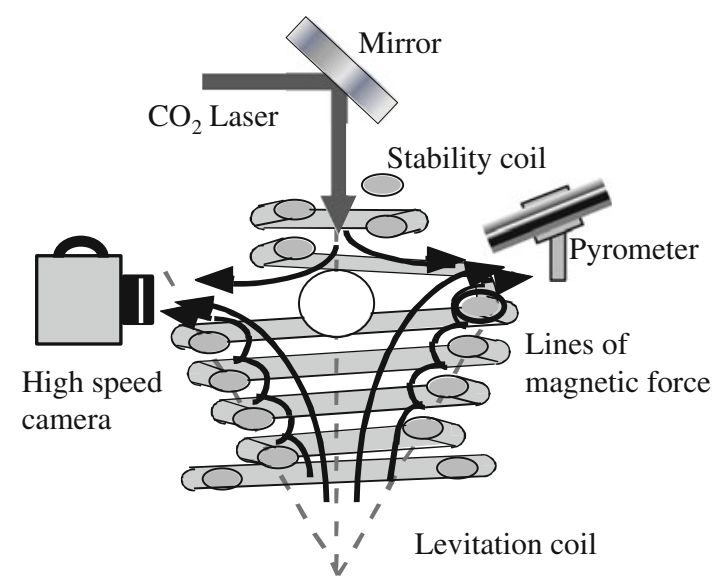

Fig. 1. Schematic illustration of the EML furnace. In order to apply EML to semiconducting material, a $\mathrm{CO}_{2}$ laser serves as a preheater.

deviated for the rate-controlling kinetics to be determined. The current investigation unifies the interpretation of containerless crystallization of semiconducting materials by expanding the analysis to include both $\mathrm{Ge}$ and $\mathrm{Si}$ to resolve remaining inconsistencies.

\section{EXPERIMENTAL PROCEDURE}

Containerless crystallization experiments on pure samples of $\mathrm{Ge}$ and $\mathrm{Si}$ were carried out by using a $\mathrm{CO}_{2}$ laser-equipped electromagnetic levitator (EML) shown schematically in Fig. 1. Except for several samples that unintentionally nucleated spontaneously, nucleation of crystals was forced by application of a trigger-needle of molybdenum at the predetermined undercooling. The growth velocity was measured using a high-speed video camera (HSV) with a maximum sampling rate of 640,000 frames/s; the Si-camera was color video, whereas the Ge-camera was monochrome. The temperature of the levitated samples was measured with a monochromatic pyrometer. Since the emissivity of the solid phase of Si and Ge greatly differs from those of the liquid phase, the emissivity was adjusted so that the temperature of the liquid phase after the recalescence indicated the equilibrium melting temperature (Fig. 2). In order to elucidate the relationship between the microstructure and the rate-determining process, the melt was dropped onto an optically polished silicon plate and then solidified under controlled temperature conditions. The resulting surface morphology was analyzed posttest with a laser-scanning microscope.

\section{RESULTS}

Figure 3 shows the selected HSV images of $\mathrm{Si}$ taken during recalescence, where $\Delta T$ is the bulk undercooling just before the recalescence. ${ }^{6}$ The dark and bright areas show the undercooled melt and the solidified region, respectively. In Fig. 3a, typical HSV images taken successively at low undercooling,
$\Delta T=37 \mathrm{~K}$, show the anisotropic line-shaped pattern that forms a circumferential ring on the surface of the sample. Note that this ring is discontinuous suggesting that the line-shaped crystal is not a "line" crystal but rather a plate-like crystalline structure that penetrates the interior of the sample. When the undercooling is larger than $100 \mathrm{~K}$, facetted dendrites appear as seen in Fig. 3b. At an intermediate undercooling, however, not only facetted dendrites but also a mixed-mode of facetted dendrites and lineshaped crystals are observed as shown in Fig. 3c. In these images, the solidification front initially advances massively, and subsequently line-shaped crystals protrude from facetted dendrites showing a mixed mode of both morphologies.

In $\mathrm{Ge}$, the morphological transition with an increase in undercooling is similar to that of $\mathrm{Si}$ : that is, at low, medium and intermediate undercoolings, plate-like needle crystals, facetted dendrites and a mixed-mode of facetted dendrites and plate-like needle crystals form, respectively, as seen in Fig. 4.

Figure 5 shows the relations between $V$ and $\Delta T$ for both $\mathrm{Si}$ and Ge. Although the growth velocities of $\mathrm{Si}$ present a curve of near-quadratic shape when plotted as a function of $\Delta T$, in Ge the relation deviates from that of Si at undercoolings larger than $100 \mathrm{~K}$. WNK reported that the Si crystal growth kinetics at low and medium undercooling is not due to interface attachment but 2-D nucleation as given by Eq. 1 .

$$
V=\beta \exp \left(-\frac{\varepsilon}{k_{\mathrm{B}} \Delta T_{\mathrm{k}}}\right)
$$

where $\beta, \varepsilon$, and $\Delta T_{\mathrm{k}}$ are the rate for atoms to be incorporated into interface, the energy barrier for nucleation and growth of a crystal, and the kinetic undercooling at the crystal-melt interface, respectively. In $\mathrm{Ge}$, on the other hand, introducing the threshold undercooling $\Delta T_{\text {th }}$, Ishibashi, Nagayama and Kuribayashi (INK) ${ }^{7}$ reported that at undercoolings larger than $100 \mathrm{~K}$ the growth velocities is well expressed by linear kinetics described by Eq. 2 .

$$
V=\mu\left(\Delta T_{\mathrm{k}}-\Delta T_{\mathrm{th}}\right)
$$

It is readily apparent that there is an inconsistency in behavior when comparing the $V$ versus $\Delta T$ of Ge to that of $\mathrm{Si}$, although both are typical semiconducting material that belong to Group IV. In order to resolve this inconsistency, it is desirable to normalize the data into dimensionless variables. Figure 6 shows the relation between the dimensionless growth velocity $\tilde{V}$ and dimensionless undercooling $\Delta \tilde{T}$ given by Eqs. 3 and 4 .

$$
\tilde{V}=\frac{V \alpha}{a}
$$

and

$$
\Delta \tilde{T}=\frac{C_{\mathrm{P}} \Delta T}{\Delta H_{\mathrm{f}}}
$$




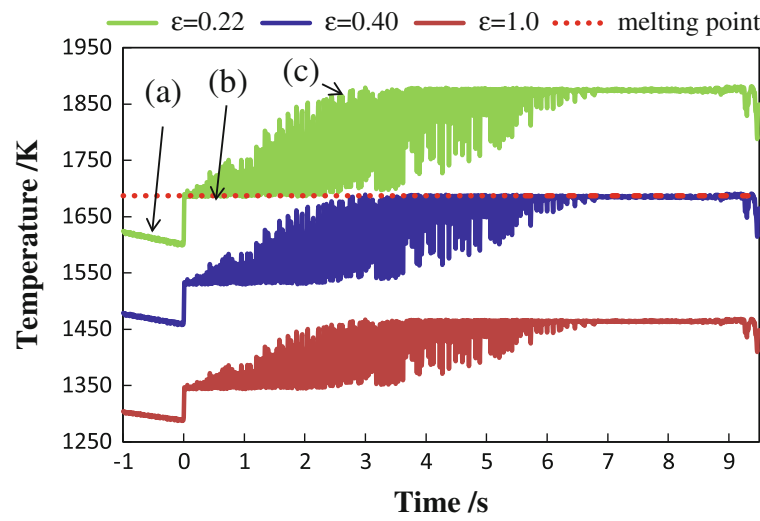

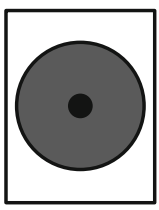

(a)

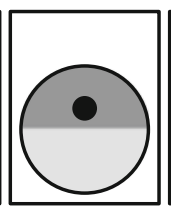

(b)

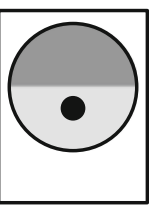

(c)

\section{undercooled liquid \\ equilibrium liquid \\ equilibrium solid \\ - measuring spot}

Fig. 2. Typical profile of temperature versus time of Si sample measured with a pyrometer. ${ }^{6}$ The temperature of the samples was determined by adjusting the emissivity so that the temperature just after the recalescence is the equilibrium melting temperature of $\mathrm{Si}$.
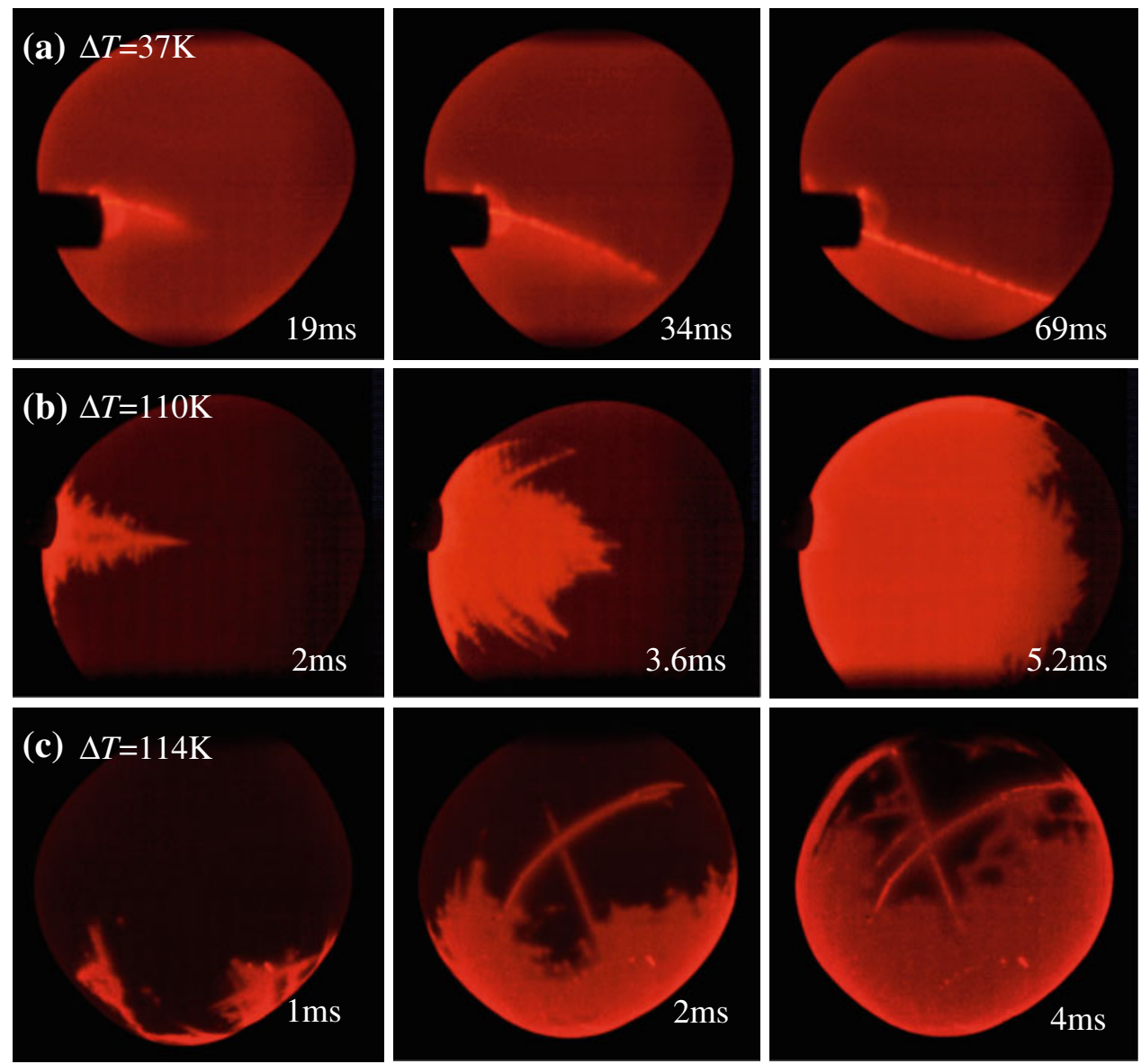

Fig. 3. HSV images of Si taken during recalescence. ${ }^{6}$ The dark and bright areas, respectively, show the undercooled melt and the solidified region. (a) HSV images taken at low undercooling, $\Delta T=37 \mathrm{~K}$, showing anisotropic line-shaped crystal that forms a circumference on the surface of the sample. (b) HSV images taken at medium undercooling, $\Delta T=114 \mathrm{~K}$, showing a facetted dendrite. (c) Mixed mode of facetted dendrites and line-shaped crystals. The solidification front initially advances massively, and subsequently line-shaped crystal protrudes from facetted dendrites showing a mixed mode of both morphologies. The growth velocity of line-shaped crystals is much higher than that of facetted dendrites.

where $\alpha, a, C_{\mathrm{P}}$, and $\Delta H_{\mathrm{f}}$, which are listed in Table I, are the thermal diffusivity, the interatomic distance, the heat capacity under the constant pressure, and the heat of fusion, respectively. Of particular importance is the observation that in regions I and II for both $\mathrm{Si}$ and Ge, crystal growth into undercooled melt can be scaled by the thermal diffusivity and the temperature increase caused by the release of latent heat, although the crystal morphology distinctly changed from the plate-like needle crystals in region I to the facetted dendrites 

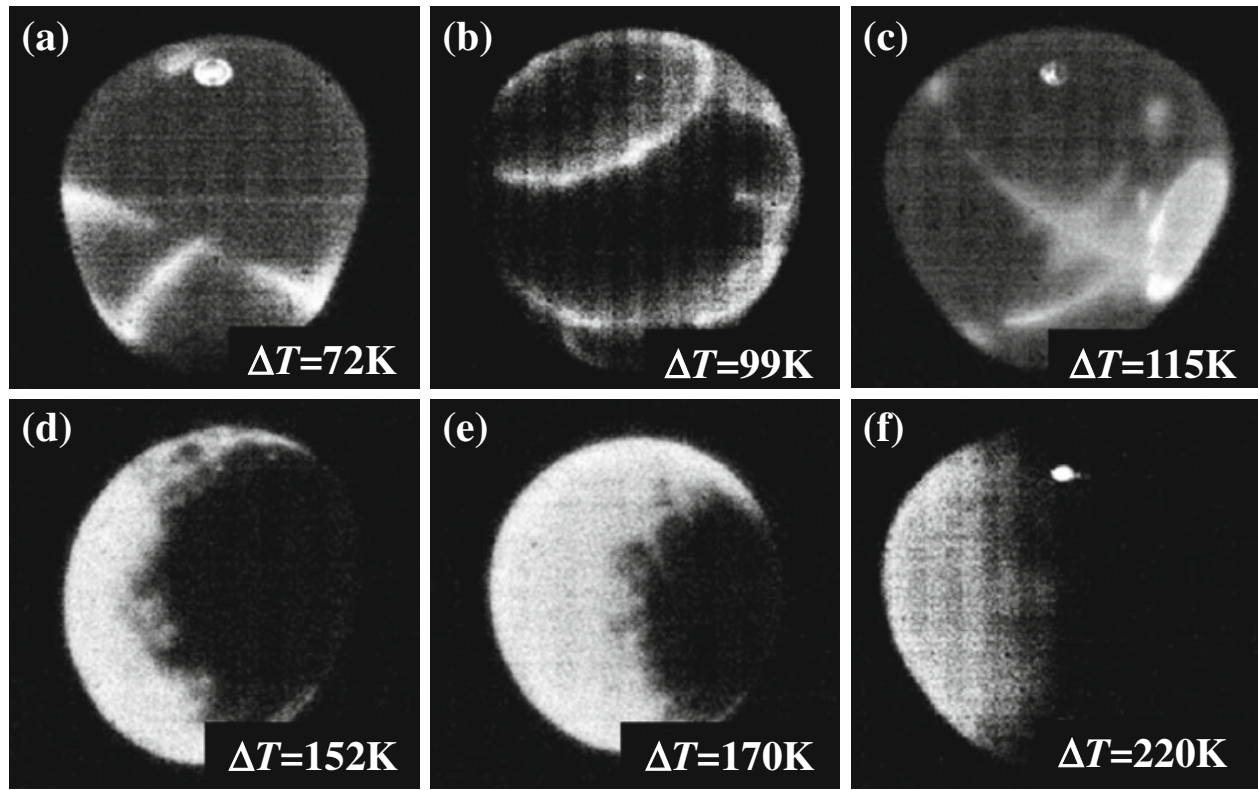

Fig. 4. HSV images of Ge taken during recalescence. ${ }^{7}$ (a) and (b) HSV images taken at low undercooling, $\Delta T=72 \mathrm{~K}$ and $99 \mathrm{~K}$. (d) and (e) HSV images taken at medium undercooling, $\Delta T=152 \mathrm{~K}$ and $170 \mathrm{~K}$. (c) Mixed mode of facetted dendrites and line-shaped crystals.

in region II. This result implies that the growth velocities in regions I and II are proportional to the product of the thermal diffusive speed $V_{\mathrm{T}}$ and the kinetics term as a function of Gibbs free energy difference between crystal and melt $\Delta G$. Therefore, if the linear approximation for the free energy difference $\Delta G=\Delta H_{\mathrm{f}} \Delta T / T_{\mathrm{m}} \quad\left(T_{\mathrm{m}}\right.$ is the equilibrium melting temperature) is used and the kinetic term is given by a linear function of the undercooling, then the relationship between the growth velocity and the undercooling is readily described by a modified Wilson-Frenkel law ${ }^{8,9}$ at low undercoolings.

$$
V \propto V_{\mathrm{T}}\left[1-\exp \left(\frac{\Delta G}{k_{\mathrm{B}} T}\right)\right]=\frac{V_{\mathrm{T}} \Delta H_{\mathrm{f}}}{k_{\mathrm{B}} T_{\mathrm{m}}^{2}} \Delta T
$$

On the morphological characterization of the growing crystal in semiconducting material, Lau and Kui, ${ }^{10}$ carrying out the quasi-containerless crystallization in Ge, in which the melt was surrounded by oxide flux, reported that the twin-related $\langle 110\rangle$ and $\langle 211\rangle$ dendrites appear at low and medium undercoolings, and the twin-free $\langle 100\rangle$ dendrites are at high undercooling $\Delta T>93 \mathrm{~K}$. Furthermore, Leung and Kui, ${ }^{11}$ extending their containerless technique to $\mathrm{Si}$, reported the similar results: at low and medium undercooling, twin-related $\langle 110\rangle$ and $\langle 211\rangle$ dendrites and at high undercooling, twin-free $\langle 100\rangle$ dendrites. Their conclusion is very clear, but the electron-micrographs that were used as the evidence for their conclusions are obscure. On this point, Nagashio and Kuribayashi, ${ }^{12}$ on the basis of scanning electron microscopy electron backscatter diffraction pattern analysis, showed that the crystallographic features of Si: $\langle 110\rangle$ dendrites are

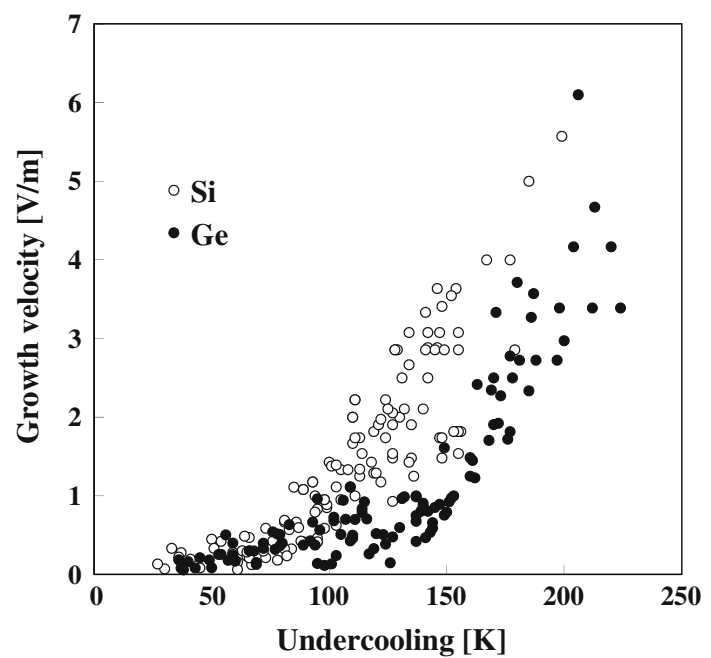

Fig. 5. Relation between growth velocity $V$ and $\Delta T$. Open and closed circles are for $\mathrm{Si}$ and $\mathrm{Ge}$, respectively.

grown under the twin-related reentrant corner mechanism, and $\langle 100\rangle$ dendrites are under the twinfree nucleation and growth mechanism. Thus, the growth directions of primary and secondary arms of the $\langle 110\rangle$ dendrites are $\langle 110\rangle$, and those of the $\langle 100\rangle$ dendrites are $\langle 100\rangle$ and $\langle 110\rangle$. On referring to their results, it appears that the plate-like needle crystal corresponds to $\langle 110\rangle$ dendrites.

Figure 7 shows the typical images of facetted dendrites of Ge: (I) solidified by dropping onto an optically polished silicon plate at $\Delta T=151 \mathrm{~K}$ showing the $\langle 100\rangle$ dendrite of four-fold symmetry, and (II) solidified at the equilibrium melting temperatures showing the twin-related $\langle 110\rangle$ dendrite. Each 


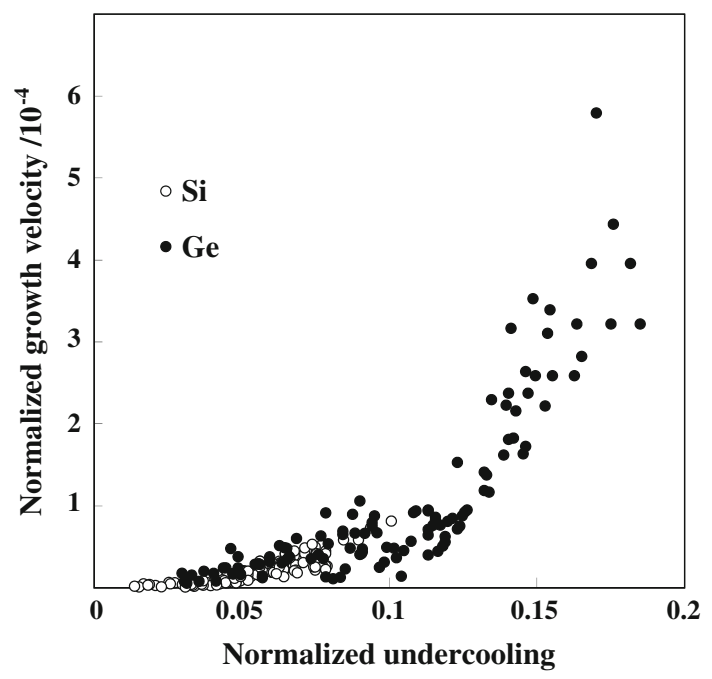

Fig. 6. Relation between dimensionless growth velocity $\tilde{V}$ and dimensionless undercooling $\Delta \tilde{T}$, which are normalized by the thermal diffusive speed $\alpha / a$ and the temperature increase due to the release of latent heat $\Delta H_{f} / C_{\mathrm{P}}$. Open and closed circles are for $\mathrm{Si}$ and Ge.

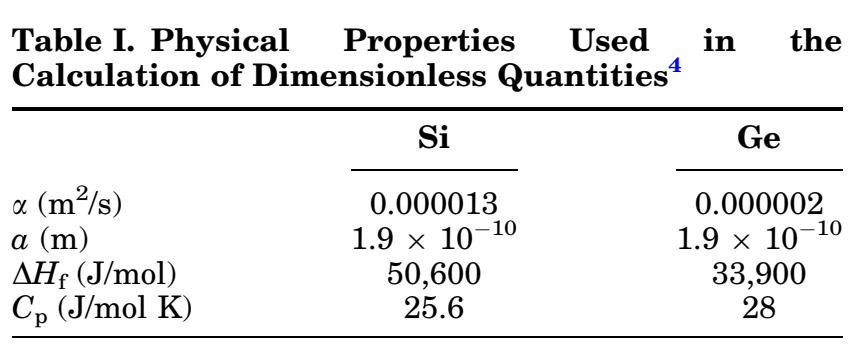

image was taken by a laser-scanning microscope. In containerless levitation experiments using a drop-quench technique, the $\langle 211\rangle$ dendrites have not been observed in Ge although Lau and Kui reported that those were formed within the range $60 \mathrm{~K}<\Delta T<90 \mathrm{~K}$. However, the morphology of the $\langle 211\rangle$ dendrite that they present is different from that of the Billig's $\langle 211\rangle$ dendrite ${ }^{13}$ and the azimuth relation between triangular etch-pit on the surface and the stem of the dendrite is also different from that of the $\langle 211\rangle$ dendrite. Furthermore, it was reported that the growth of the $\langle 211\rangle$ dendrite would be caused by an impurity effect due to the presence of the oxide flux. ${ }^{14}$ For formation of $\langle 211\rangle$ dendrites some geometrical restrictions such as unidirectional pulling at very low undercooling are required because multiple interactions among 2-D nuclei on three adjoining reentrant corners are required for the $\langle 211\rangle$ dendrite to be formed. For the $\langle 110\rangle$ dendrites only, a single interaction between 2-D nuclei is required on adjacent reentrant corners. ${ }^{15}$ Finally, in $\mathrm{Si}$ and $\mathrm{Ge}$, the direction of the interface stiffness minimum corresponds to that of the interface free energy maximum, that is, $\langle 100\rangle,{ }^{16}$ and dendrites are known to grow preferentially in the direction of the interface stiffness minimum.

\section{DISCUSSION}

As mentioned above, WNK reported that the ratecontrolling kinetics for crystal growth in regions I and II for $\mathrm{Si}$ is not linear, which would imply interface attachment kinetics, but rather exponential in character, which would indicate 2-D nucleation and growth is controlling. INK also reported the similar result in Ge. Based on these results, the growth velocities of semiconducting material previously expressed in Eq. 1 can be modified to a new form as seen in Eq. 6.

$$
V=\beta^{\prime} \alpha \exp \left(-\frac{\varepsilon}{k_{\mathrm{B}} \Delta T_{\mathrm{k}}}\right)
$$

where $\beta^{\prime}$ is the adhesive probability for atoms that are adhered on the surface to be incorporated into the crystal lattice. In fact, the relation between the growth velocity and the undercooling in Ge, which was presented by Hamilton and Seidensticker, ${ }^{17}$ implied that the crystal growth kinetics is not due to linear kinetics because if the crystal growth is expressed in this manner, the growth velocity is inversely proportional to the spacing between two parallel twins (see Appendix).

At region III, which corresponds to the undercooling larger than approximately $100 \mathrm{~K}$ in Ge and $200 \mathrm{~K}$ in $\mathrm{Si}$, the growth kinetics is seen to be the linear in character. Figure 8 shows a micrograph of a section of Si solidified at $\Delta T=292 \mathrm{~K} .{ }^{18} \mathrm{In}$ this experiment, a small amount of boron was doped to accentuate the contrast of the interface of the initially formed dendrite. In this figure, cross-like morphologies show the fragments broken up from the initially formed dendrite due to the capillary force that acts on the interface. The fourfold axial symmetry of the fragments indicates that the initial growth direction indicates formation of $\langle 100\rangle$ dendrites. Nagashio et al. ${ }^{18}$ reported that in $\mathrm{Si}$ when the undercooling exceeds $100 \mathrm{~K}$, fragmentation of dendrites occurs resulting in significant grain refinement with increase of undercooling until $\Delta T>200 \mathrm{~K}$ when grain size becomes relatively constant. This effect is due to the cross-like fragments acting as a "seed" for new grains. If the surfaces of the primary and secondary dendrite arms are assumed to consist of $\{111\}$ planes, then the interfacial energy of which is minimum, the surface morphology of the $\langle 100\rangle$ dendrite arms should appear as a wavy pattern that consists of paired chevron and groove couples, whereas that of $\langle 110\rangle$ dendrite is flat and parallel to the dendrite axis. Therefore, in $\langle 100\rangle$ dendrites, the arms characterized by the wavy pattern are easily broken. At undercoolings higher than this, dendrite breakup will occur and observed growth velocities would deviate downward from the prediction using the dendrite growth model, due to the interference caused by the overlapping diffusion fields built up around the fragmented dendrites. Thus, the apparent diffusion length $d_{\mathrm{d}}=\alpha / V$ is elongated. Furthermore, electro-magnetic stirring facilitates dendrite frag- 

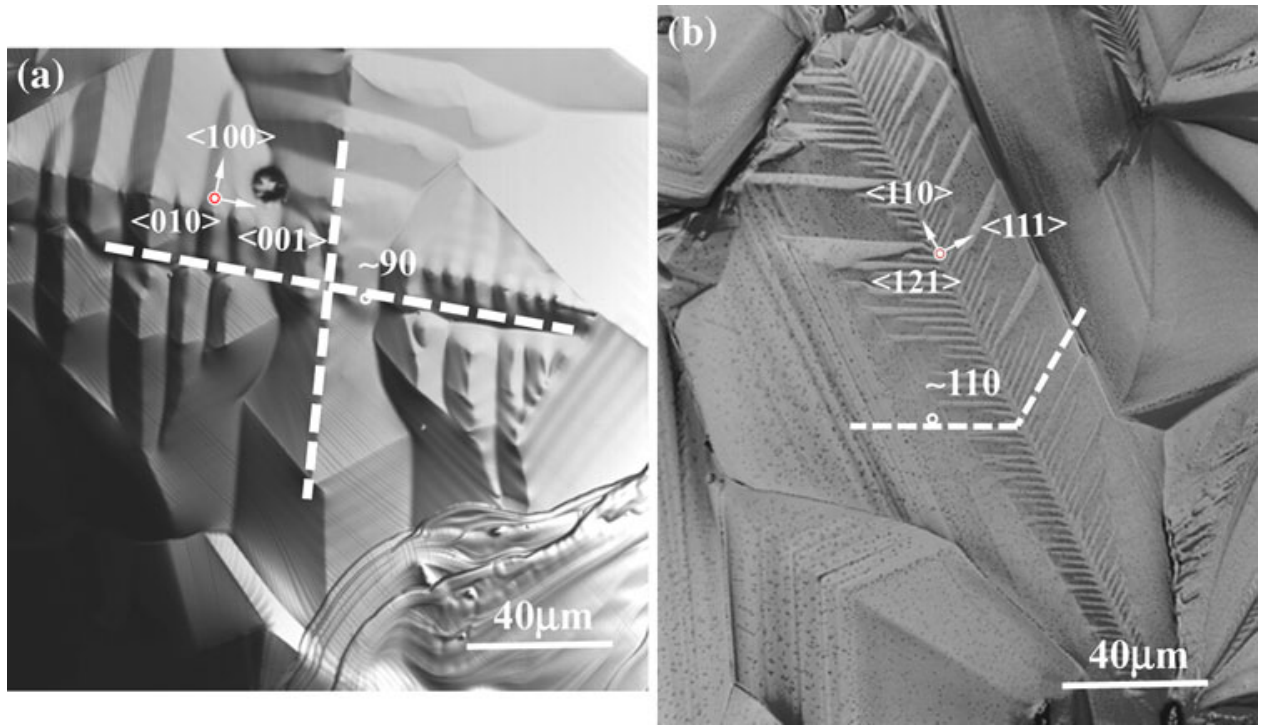

Fig. 7. Typical images of facetted dendrites in Ge samples: (a) containerlessly solidified at $\Delta T=151 \mathrm{~K}$ showing $\langle 100\rangle$ dendrite and (b) solidified by dropping onto the optically polished silicon plate at the equilibrium melting temperatures showing $\langle 110\rangle$ dendrite. Each image was taken by laser scanning microscopy. ${ }^{7}$
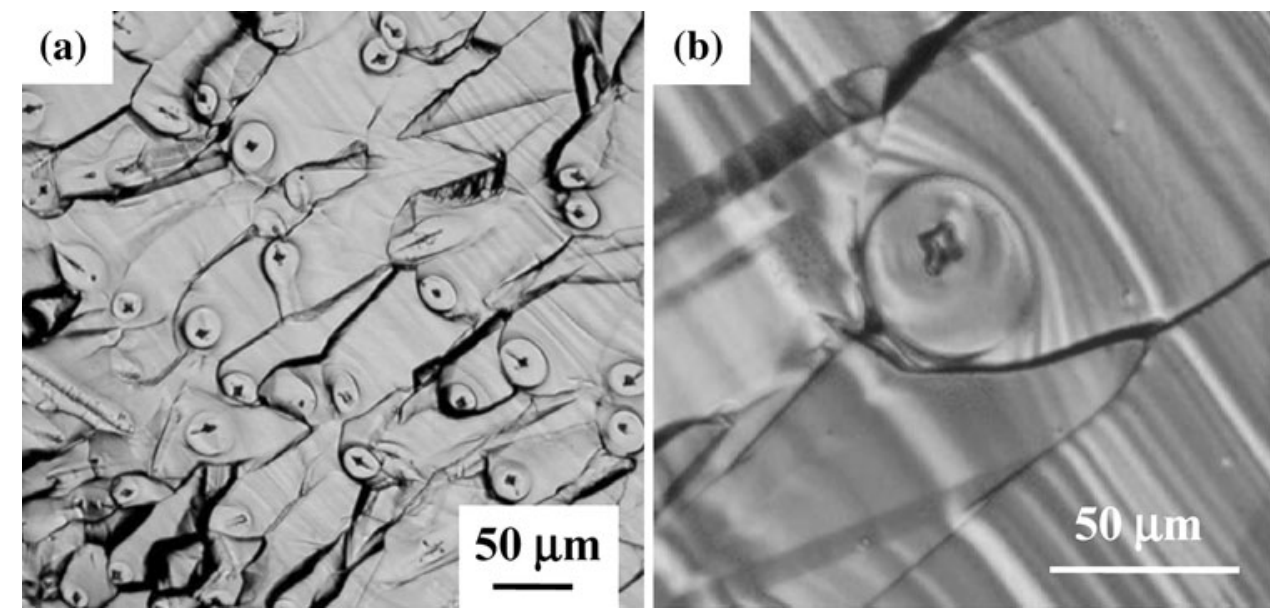

Fig. 8. (a) Optical micrograph of the cross section of the Si sample solidified at $\Delta T=292 \mathrm{~K}$. (b) Optical micrograph of the magnified region of a typical fragmented cross-like dendrite. ${ }^{18}$

mentation. ${ }^{19}$ On the other hand, Devaud and Turnbull, ${ }^{20}$ and Lau and Kui reported that in Ge the grain refinement occurred at $\Delta T>300 \mathrm{~K}$ and $\Delta T \sim 230 \mathrm{~K}$. This is because for Ge the value of $\tilde{V}$ at $\Delta \tilde{T}>0.15$ differs from that of Si.

In the previous section, we showed that the crystal growth velocities at regions I and II in both $\mathrm{Si}$ and $\mathrm{Ge}$ are expressed by the thermal diffusioncontrolled growth model. It is common to evaluate growth of other nonsemiconductor pure materials using the "Collision-limited growth model." reason for this is that in the atomic diffusion-controlled growth model, a diffusive velocity given by $V_{\mathrm{D}}=D / a(D$ is the self-diffusion coefficient) is at most $10-20 \mathrm{~m} / \mathrm{s}$, which is much less than the growth velocities measured in pure Ni samples. ${ }^{22}$ However, the growth velocity predicted by the collisionlimited growth model is much larger than that simulated by molecular dynamics and densityfunctional theory. ${ }^{23}$ We must use the diffusioncontrolled growth model because for semiconductors, the larger entropy difference between the melt and the crystal requires a massive scale of the thermal diffusion from the interface to the undercooled melt.

\section{CONCLUSION}

Using $\mathrm{Si}$ and $\mathrm{Ge}$ as the model material, we carried out the undercooled solidification of the semiconducting material. From the point of the interface morphologies, the relation between growth velocities 
and undercoolings was classified into three regions. The morphologies of the growing crystals at regions I, II, and III are plate-like needle crystals, facetted dendrites, and massive dendrites, respectively. Normalization of the relation between growth velocities and undercoolings into dimensionless quantities revealed that the growth velocities in regions I and II can be scaled by the thermal diffusivity and the temperature increase caused by the release of the latent heat, indicating that the growth velocities are proportional to the product of the thermal diffusive speed and the kinetics term as a function of Gibbs free energy difference. Analyzing the growth velocities on the basis of this dendrite growth model, it was found that at low undercooling, the rate-controlling process is two-dimensional nucleation kinetics, and at high undercooling thermal diffusion-controlled interface attachment kinetics.

\section{OPEN ACCESS}

This article is distributed under the terms of the Creative Commons Attribution License which permits any use, distribution, and reproduction in any medium, provided the original author(s) and the source are credited.

\section{APPENDIX}

If the tip of a growing crystal can be assumed to be hemispherical in shape, then the diffusion equation at the steady state for heat conduction into undercooled melt is expressed by

$$
\frac{\mathrm{d}^{2} T(r)}{\mathrm{d} r^{2}}+\frac{2}{r} \frac{\mathrm{d} T(r)}{\mathrm{d} r}=0
$$

where $T(r)$ is the temperature at $r$, the distance from the centroid of the tip. If the boundary condition is given by $T=T_{\infty}$ at $r=\infty$, then $T(r)$ is expressed by

$$
T(r)=\frac{A}{r}+T_{\infty}
$$

If the growth velocity is assumed to be proportional to the temperature gradient and also undercooling at the crystal-melt interface, $r=r_{i}$, then the growth velocity is given by

$$
\begin{aligned}
V=-\left.\frac{C_{\mathrm{P}} \alpha}{\Delta H_{\mathrm{f}}} \frac{\mathrm{d} T}{\mathrm{~d} r}\right|_{r=r_{i}} & =-\frac{C_{\mathrm{P}} \alpha}{\Delta H_{f}} \frac{A}{r_{i}^{2}} \\
& =\mu\left\lfloor\left(T_{\mathrm{m}}-\frac{2 \Gamma}{r_{i}}\right)-\left(T_{\infty}+\frac{A}{r_{i}}\right)\right\rfloor
\end{aligned}
$$

Therefore the constant of integration $A$ is expressed by

$$
A=\frac{r_{i}\left(T_{\mathrm{m}}-T_{\infty}-\frac{2 \Gamma}{r_{i}}\right)}{l_{k}+r_{i}}
$$

where $l_{k}$ is a kinetics length given by $l_{k}=C_{\mathrm{P}} \alpha / \mu \Delta H_{\mathrm{f}}$ and $\Gamma$ is Gibbs-Thomson coefficient $\Gamma=\gamma / \Delta S_{\mathrm{f}}$. Then the thermal diffusion-controlled crystal growth velocity into the undercooled melt is given by

$$
\tilde{V}=\frac{a \Delta \tilde{T}_{k}}{r_{i}+l_{k}}\left(1-\frac{r_{n}^{*}}{r_{i}}\right)
$$

where $r_{i}, l_{k}$, and $r_{n}^{*}$ are the tip radius, kinetic length given by $r_{n}^{*}=2 \gamma / \Delta S_{\mathrm{f}} \Delta T_{k}$.

In the case of the crystal growth based on the twinrelated reentrant corner growth model, $r_{i}$ can be assumed to be less than the spacing between two parallel twins. Therefore, Eq. A5 can be approximated by

$$
\tilde{V} \approx \frac{a \Delta \tilde{T}_{k}}{r_{i}}
$$

because $r_{i} \gg l_{k}$ and $r_{i} \gg r_{n}^{*}$. Thus, the growth velocity is inversely proportional to the spacing between two parallel twins.

\section{REFERENCES}

1. D.M. Herlach, Mater. Sci. Eng. R12, 177 (1994).

2. D.M. Herlach, P.G. Galenko, and D. Holand-Moritz, Metastable Solids from Undercooled Melts, ed. R. Cahn (Amsterdam, the Netherlands: Elsevier, 2007), p. 1.

3. W.R. McKee, IEEE Trans. Compon. Hybrids Manuf. Technol. 5, 336 (1982).

4. T. Aoyama, Y. Takamura, and K. Kuribayashi, Metall. Mater. Trans. A 30A, 1333 (1999).

5. J. Lipton, W. Kurz, and R. Trivedi, Acta Metall. 35, 957 (1987).

6. K. Watanabe, K. Nagayama, and K. Kuribayashi, J. Phys. Conf. Ser. 327, 012018 (2011).

7. Y. Ishibasi, K. Nagayama, and K. Kuribayashi, J. Japan Inst. Metals 76, 380 (2012).

8. Y. Saito, Statistical Physics of Crystal Growth (Singapore: World Scientific, 1996), p. 65.

9. D.Y. Sun, M. Asta, and J.J. Hoyt, Phys. Rev. B 69, 024108 (2004).

10. C.F. Lau and H.K. Kui, Acta Metall. Mater. 41, 1999 (1993).

11. K.K. Leung and H.W. Kui, J. Appl. Phys. 75, 1216 (1994).

12. K. Nagashio and K. Kuribayashi, Acta Mater. 53, 3021 (2005).

13. E. Billig, Proc. R. Soc. Lond. A 229, 346 (1955).

14. S. O'Hara, Metallurgy of Elemental and Compound Semiconductors, ed. R.O. Grubel (New York: Interscience Publisher, 1961), pp. 149-160.

15. K. Fujiwara, H. Fukuda, N. Usami, K. Nakajima, and S. Uda, Phys. Rev. B 81, 224106 (2010).

16. P.A. Apte and X.C. Zeng, Appl. Phys. Lett. 92, 221903 (2008).

17. D.R. Hamilton and R.G. Seidensticker, J. Appl. Phys. 34, 1450 (1963).

18. K. Nagashio, H. Okamoto, K. Kuribayashi, and I. Jimbo, Metall. Mater. Trans. A 36A, 3407 (2005).

19. H. Yasuda, I. Ohnaka, Y. Ninomiya, R. Ishii, S. Fujita, and K. Kishio, J. Cryst. Growth 260, 475 (2004).

20. G. Devaud and D. Turnbull, Acta Metall. 35, 765 (1987).

21. C.A. Donald, M.A. Malvezzi, and F. Spaepen, J. Appl. Phys. 65, 129 (1989).

22. E. Eckler and D.M. Herlach, Mater. Sci. Eng. A 178, 159 (1994).

23. J.J. Hoyt, M. Asta, T. Haxhimali, A. Karma, R.E. Napolitano, R. Trivedi, B.B. Laird, and J.R. Morris, MRS Bull. Dec., 935 (2004). 